\title{
Mate Availability and Sexual Disgust
}

\author{
Courtney L. Crosby ${ }^{1}$ (D) Patrick K. Durkee ${ }^{1}$ - Anna G. B. Sedlacek ${ }^{1}$. \\ David M. Buss ${ }^{1}$
}

Received: 5 February 2020 / Revised: 23 April 2021 / Accepted: 28 April 2021 / Published online: 13 May 2021 (c) The Author(s), under exclusive licence to Springer Nature Switzerland AG 2021

\begin{abstract}
Objective One of the factors that sexual disgust should be calibrated to is the size of the mating pool. This study tested this hypothesis by examining whether perceptions of mate availability explain variance in levels of sexual disgust towards potential mates.
\end{abstract}

Methods Participants ( $N=853 ; 373$ women) rated how sexually disgusting they found 60 potential mates that have previously been rated on attractiveness by a separate group of raters. We also measured participants' perceptions of mate availability in their local environment, self-perceived attractiveness and mate value, and relevant control variables.

Results Multilevel models revealed a negative association between sexual disgust towards potential mates and perceived mate availability- the opposite of what we predicted. We found support for our prediction that women had higher levels of sexual disgust than men, but only after addressing the confounding sex difference in target attractiveness. We also found the predicted negative association between target attractiveness and sexual disgust. Finally, as predicted, sexual disgust levels were more strongly related to potential mates' attractiveness in individuals who perceived there to be many available mates in their local environment.

Conclusions These findings generally bolster functional accounts of sexual disgust while highlighting the need for more evidence to ascertain the role of mate availability in the calibration of sexual disgust. Specifically, future research should examine the extent to which disgust levels may truncate mental representations of the mating pool instead of being calibrated by them.

Keywords Sexual disgust $\cdot$ Mate availability $\cdot$ Attractiveness $\cdot$ Mating $\cdot$ Evolution

Patrick K. Durkee contributed equally to this work and shares first authorship.

Courtney L. Crosby

clcrosby@utexas.edu

1 Department of Psychology, The University of Texas At Austin, 108 E. Dean Keeton St, Austin, TX 78712, USA 


\section{Mate Availability and Sexual Disgust}

Why and when do individuals experience sexual disgust? Evolutionary scientists have begun to develop an understanding of the functional role of sexual disgust. Sexual disgust is hypothesized to be an emotion that has been shaped by selection pressures that are partially distinct from other forms of disgust, such as pathogen disgust and moral disgust (Crosby et al., 2020; Curtis et al., 2004; Tybur, et al., 2009). A central assumption underlying this hypothesis is that by experiencing sexual disgust in specific sexual contexts, individuals are guided away from mating opportunities that would have, on average, led to lower reproductive fitness over the course of human evolution.

As with other emotions, sexual disgust is hypothesized to consist of a computational architecture wherein specific inputs trigger its activation (Al-Shawaf et al., 2016; Cosmides \& Tooby, 2000; Nesse, 1990; Tybur et al., 2013). Once activated, sexual disgust should direct individuals away from potentially costly mating opportunities or sub-optimal mates, such as genetic relatives (Ackerman et al., 2007; Haidt et al., 2000) or mates with cues to communicable diseases (Ryan et al., 2012). Given the range of cues that are probabilistically associated with the presence of potential fitness costs in sexual domains (e.g., disease cues, investment cues), sexual disgust should be sensitive to multiple, predictable inputs including situational contexts and individual traits (Tybur et al., 2013).

One of the most salient environmental factors to which sexual disgust should be calibrated is the local mating pool (Lieberman \& Patrick, 2018; Tybur et al., 2013). Though the adaptive problem of selecting a high-quality mate is incredibly important, the potential benefits of being highly selective become outweighed by the costs associated with not reproducing as the number of mating options dwindles (Daly \& Wilson, 2001). Consequently, perceptions of potential mates are hypothesized to be calibrated to the size of the mating pool. A relative dearth of available mates is predicted to lower sexual disgust levels and motivate approach behavior to increase the odds of mating success. Conversely, sexual disgust should be higher in a more densely populated mating pool, increasing selectivity ${ }^{1}$.

While mate availability perceptions should track the true mate availability in the local environment, the estimation may not be perfectly accurate. The inputs into mechanisms facilitating mate choice can only be mental estimations: there is no direct sensory stimulus that can adequately simulate the size and makeup of all the individuals in a local mating pool. Thus, if mate availability drives downstream behavioral changes through sexual disgust, disgust levels should be calibrated as a function of perceived mate availability more than any objective measure. For example, if an individual perceives that there are few available mates to select from and

\footnotetext{
1 Before, we discussed disgust activation using thresholds, saying "A relative dearth of available mates is predicted to raise the threshold for sexual disgust and motivate approach behavior to increase the odds of mating success. Conversely, the threshold for sexual disgust is expected to be lower in a more densely populated mating pool, increasing selectivity." We changed the language from thresholds to sexual disgust levels to be consistent and to ease readership. This did not alter our original hypothesis
} 
that these circumstances are unlikely to change, the expected sexual value of the mates that are available should be higher.

While the relationship between mate availability and sexual disgust has not been directly examined in the literature, there is empirical support for the impact of mate availability on a number of related constructs. Prior studies have attempted to experimentally manipulate perceptions of mate availability with text-based information and edited photos. For example, Griskevicius et al. (2012) presented photos of varying numbers of men and women and found that a male-biased sex ratio (i.e., a scarcity of women) resulted in an expectation for men to spend more money during courtship. Other researchers have fabricated newspaper articles about sex-ratio imbalances (Arnocky et al., 2014, 2016; Hahn et al., 2014; Watkins et al., 2012). For example, Arnocky et al. (2016) presented participants with one of two conditions: one priming mate scarcity or another priming mate abundance. The study found that heterosexual men, but not women, in the mate abundance condition had higher infidelity intentions and less restricted sociosexuality.

Despite these findings, there are reasons to be skeptical about the validity of manipulating mate availability perceptions within a lab setting. While it is possible that these evolutionarily-novel stimuli may serve as inputs into our evolved mate selection mechanisms, natural selection likely favored perception psychologies that make accurate and robust inferences about the relative number of available men and women in an individual's local ecology. There are possible costs to allowing novel, potentially unreliable information to sway perceptions of the mating pool: others might exploit this heuristic in favor of their own mating strategy, or an individual's mating strategy could be easily miscalibrated. A more ecologically valid way of assessing the potential influence of mate availability on sexual disgust may be to measure individuals' perceptions of mate availability in their own ecology to examine the influence of this internal regulatory variable on sexual disgust parameters.

In the current study, we aimed to more directly measure internal estimations of mate availability to test the hypothesis that perceived mate availability is positively associated with baseline levels of sexual disgust across potential mates (H1). That is, we hypothesized that people who perceived there to be low mate availability in their current environment would consider novel, potential mates as less sexually disgusting, on average, than people who perceived there to be high mate availability in their current environment. The influence of mate availability perceptions on disgust levels may be subtle, so we conservatively predicted that this relationship would be small.

We also tested three additional peripheral hypotheses about sexual disgust. Because men tend to be less disgusted than women, on average (Al-Shawaf et al., 2018; Crosby et al., 2020; Tybur et al., 2009), we predicted that men's baseline sexual disgust levels across potential mates would be lower than women's baseline sexual disgust $(\mathrm{H} 2)$. Based on previous research, we expected this sex difference to be fairly large (Al-Shawaf et al., 2018; Al-Shawaf et al., 2015; Crosby et al., 2020; Tybur et al., 2009).

Additionally, as a composite of many fitness-relevant cues, facial attractiveness is component of overall mate-value (Fisher et al., 2008; Little et al., 2011; Thornhill \& Gangestad, 1999) — which is another input hypothesized to calibrate sexual disgust levels (Tybur et al., 2013). Thus, we hypothesized that sexual 
disgust would be negatively associated with potential mates' facial attractiveness such that more attractive mates would be considered less sexually disgusting (H3). Given the relative importance of physical attractiveness in mating decisions (Buss, 1989), we expected this association to be large.

Finally, we examined whether mate-availability estimations moderated the association between potential mates' facial attractiveness and an individual's sexual disgust. Specifically, we predicted that sexual disgust should be more dependent on the physical attractiveness of potential mates for individuals that have an internal representation that there are many available mates in the environment than for individuals that perceive there to be few available mates in the environment (H4). More plainly stated, if an individual perceives there to be many mates to select from, the attractiveness of potential mates should be weighted more heavily, and individuals should be even more disgusted by less-attractive individuals. We expected this interaction effect to be small.

To test these hypotheses, we asked a sample of raters to indicate their level of sexual disgust towards potential mates. Raters were also asked to report perceptions of mate availability within their environment. The stimuli that we presented to raters as potential mates have been previously rated on attractiveness by raters from 11 world regions (Jones et al., 2018), providing a somewhat objective estimation of each potential mate's attractiveness across environments. We used these average ratings to examine how general facial attractiveness-which itself is a composite of various cues to fitness relevant information (Little et al., 2011; Thornhill \& Gangestad, 1999)_relates to sexual disgust levels.

To more accurately test our hypotheses, we controlled for several potentially confounding factors. First, perceptions of available mates and sexual disgust levels may both be influenced by an individual's self-perceived mate-value and attractiveness. For example, more attractive, higher mate-value individuals tend to be more selective on average than less attractive, lower mate-value individuals (Arnocky, 2018; Buss \& Shackelford, 2008; Yong \& Li, 2012). As a result, attractive individuals may even perceive there to be fewer desirable mates to select from in their environment. Second, an individual's current relationship status may also confound perceptions of available mates and sexual disgust levels towards potential mates other than their relationship partner. Whether individuals are pursuing short- or long-term relationships may similarly alter mate-availability perceptions and disgust levels if, for instance, short-term oriented people perceive there to be more desirable mates around (Al-Shawaf et al., 2019; Crosby et al., 2020; Tybur et al., 2009). Because each of these factors could have confounded our measurement of participants' mate availability perceptions, we controlled for these and other nuisance variables (e.g., rater age, ethnicity) in our analyses in order to more precisely assess the unique association between mate availability perceptions and sexual disgust levels. Finally, because the nuances of long-term and short-term mating contexts may complicate the hypothesized relationships between sexual disgust and mate availability perceptions (Buss \& Schmitt, 1993), we focused only on short-term mating decisions in our study. 


\section{Method}

\section{Participants}

Participants were recruited from Prolific, the psychology subject pool at our University, and snowball sampling through social media platforms (e.g., Facebook, Twitter, Reddit) to complete the study. Participants recruited through Prolific were paid $\$ 1.09^{2}$ to complete the survey. To participate, we required Prolific users to have a minimum approval rating of $90 \% .{ }^{3}$ Participants recruited through our University's psychology subject pool were compensated with 0.50 hours of course credit, and participants recruited through social media platforms were not compensated. We had separate survey links for these three sources of recruitment. The survey itself was identical across platforms. All surveys were launched at the same time. Prior to data analysis, we merged these three datasets. We had enough funds to recruit $1,151^{4}$ participants from Prolific and we recruited the balance of our sample via snowball sampling and the department's subject pool. All study procedures were IRB approved.

Our target sample size was based on power simulations we conducted (see Power Simulations code: https://osf.io/vcu9r/?view_only=3dd4467ac8584d6b9c561a5a0 39f774e) prior to beginning data collection. Given the importance of sexual disgust for mating decisions, it is likely that even a very small relationship between perceptions of available mates and disgust levels would be theoretically meaningful. We estimated that we needed around 900 raters to achieve $90 \%$ power to detect our smallest hypothesized effect (i.e., the interaction between mate availability perceptions and attractiveness of potential mates) with our fixed number of stimuli (i.e., 120 faces).

We recruited men and women between the ages of 18 and 80 . We recruited a larger number of participants upfront to ensure that we had an adequate sample size after excluding participants based on our exclusion criteria. We also monitored the sample sizes periodically as we collected data to ensure that we got as close to our proposed sample size as possible. Our sample size before exclusions was 2,831 ( $n=1,239$ men, 1,517 women, and 12 participants who identified as "other"). Unfortunately, we lost a large portion of participants to our preregistered exclusion criteria ${ }^{5}$ : failed attention

\footnotetext{
2 We originally planned on compensating participants $\$ 1.00$, but had to change the amount to $\$ 1.09$ by the time we launched the survey due to Prolific's compensation policies.

3 We started out requiring an approval rate of $80 \%$ for Prolific participants. However, about halfway through data collection, we noticed that we were losing a portion of Prolific participants to failed attention checks. To try to prevent further participant attrition, we increased the minimum approval rating to $90 \%$ and started only compensating individuals who passed the attention checks.

4 The third author on this paper received an emergency bridging grant from the Society for Personality and Social Psychology (SPSP) to fund recruitment for this study. As a result, we had more funds to compensate participants than we originally proposed.

5 It is unclear why we lost so many participants to failed attention checks, careless responding, or validity questions. One reason may be because we initially proposed compensating participants recruited through Prolific for completing the study regardless of their responses to attention checks, careless responding, or validity questions. After changing the recruitment procedure, however, we experienced a decline in failed attention checks. For university students, we compensated participants regardless of their responses to attention checks. We lost 646 university participants to failed attention checks, sexual orientation, or validity questions. Future researchers may consider using more stringent recruitment requirements to prevent participant attrition.
} 
checks $(n=767)$, careless responding $(n=577)$, being non-heterosexual $(n=240)$, reporting that they were not paying attention and responding honestly to at least $80 \%$ of the survey $(n=201)$, being under $18(n=177)$, or being heterosexual but indicating a sexual preference for members of the same-sex $(n=3)$. Our final sample size consisted of 853 participants (480 male). Of our final participants, 518 were recruited from Prolific, 144 participants were recruited from snowballing through social media websites, and 191 participants were recruited from our university's subject pool. Of our final sample, the majority of our participants identified as white (70.34\%) and all were heterosexual. The average time it took to complete the survey for our final sample was $15.39 \mathrm{~min}$. Access all of our raw, anonymized data files by visiting: https://osf.io/vcu9r/?view_only=3dd44 67ac8584d6b9c561a5a039f774e.

\section{Materials}

The complete Qualtrics survey depicting all questions asked, stimuli presented, and survey logic is available on the Open Science Framework (https://osf.io/vcu9r/?view_ only $=3 \mathrm{dd} 4467 \mathrm{ac} 8584 \mathrm{~d} 6 \mathrm{~b} 9 \mathrm{c} 561 \mathrm{a} 5 \mathrm{a} 039 \mathrm{f} 774 \mathrm{e})$. The survey can be viewed using this preview link: https://utexas.qualtrics.com/jfe/form/SV_4MczblNwFODFEdo.

Face stimuli. We used 120 photos of men's $(n=60)$ and women's $(n=60)$ faces from the Chicago Face Database (Ma et al., 2015) that were used in Jones et al. (2018) as stimuli in our study. This stimulus set contained photographs of 30 White (15 male), 30 Asian (15 male), 30 black (15 male), and 30 Latin (15 male) faces. The average age of the stimulus subjects was 26.40 years $(S D=3.60)$. Each photo had already been rated on 13 social perception dimensions by raters across 11 world regions as part of Jones et al. (2018). We used the third-party attractiveness ratings aggregated across a random subset $(N=3,851)$ of raters from all 11 world regions in our analyses as a somewhat "objective" measure of targets' physical attractiveness across human environments (https://osf.io/ufnm6/).

Demographics. We asked participants to report their age, ethnicity, sex assigned at birth, gender identity, sexual orientation, and gender they were most sexually attracted to (see https://osf.io/vcu9r/?view_only=3dd4467ac8584d6b9c561a5a039f774e for all study questionnaires).

Perception of available mates. We developed four carefully worded items to assess participants' perceptions of the size of the mating pool in their local environment without focusing on the number of people participants would or could actually mate with: (1) "There are a lot of people of the gender that I am attracted to who are available to date casually in my area (regardless of their interest in me or my attraction to them)"; (2) "There are a lot of people of the gender that I am attracted to who are sexually available in my area (regardless of their interest in me or my attraction to them)"; (3) "There are more people in my area who are sexually available than who are sexually unavailable (regardless of their interest in me or my attraction to them)"; and (4) "There are not a lot of people of the 
gender that I am attracted to in my area who are sexually available (reverse coded)". Participants indicated the degree to which they agree with each statement using a four-point scale ( $1=$ strongly disagree; $2=$ somewhat disagree; $3=$ somewhat agree; $4=$ strongly agree). The reliability of this self-perceived mate availability scale was adequate (Cronbach's $\alpha=$ 0.82 ; Guttman's $\lambda 2=0.79$ ).

Sexual disgust. We asked participants to report their level of sexual disgust towards each face by asking "How grossed out are you by the thought of having a short-term sexual encounter with this person (e.g., intercourse; hookup; one night stand)?" on a scale of 1 (not at all grossed out) to 5 (extremely grossed out). We opted to use the term "grossed out" instead of "sexually disgusted" because the latter may prime more of a moral response than the former; we wanted to curtail the potential effect that participants' moral views toward short-term sexual encounters might have on their disgust ratings.

Self-perceived mate value. Following Arnocky (2018), we assessed each individual's self-perceived mate value by asking participants to fill out a modified version of the Components of Mate Value Survey (CMVS; Fisher et al., 2008). This scale is based on an earlier scale (the Self-Perceived Mating Success Scale; Landolt et al., 1995) and assesses an individual's perception of how the opposite sex views them as a potential relationship partner. We altered the questions to be more applicable to non-heterosexual participants by changing the phrase "opposite sex" to "gender I prefer to date" throughout (e.g., "members of the gender I prefer to date like me back.") the questionnaire. To lessen the burden on participants, our modified version contained only seven of the original 22 items. We removed items that were not directly related to participants' perceptions of others' interest in them as a mate (e.g., "I am popular"; "I want to have children in my lifetime") and items that could be tapping into perceived mate availability or a participants' mating self-esteem (e.g., "I often worry about not having a date"; "I would like members of the opposite sex to hit on me more than they do"). The reliability of this self-perceived mate value scale was adequate (Cronbach's $\alpha=0.90$; Guttman's $\lambda 2=0.90)$.

Self-perceived physical attractiveness. We also asked participants to rate how attractive they perceive themselves to be by asking them the extent to which they agree ( $1=$ strongly disagree; 2 =disagree; $3=$ agree; $4=$ strongly agree) with three statements about their physical attractiveness ("Compared with people my age, I have an attractive body"; "Compared with people my age, I have an attractive face"; "Compared with people my age, I am physically attractive"). The reliability of this self-perceived attractiveness scale was adequate (Cronbach's $\alpha=0.82$; Guttman's $\lambda 2=0.78$ ).

Current relationship status and mating orientation. In this study, we controlled for relationship status and mating orientation. Being in a relationship or being highly motivated towards short-term mating may influence disgust such that these individuals may be more or less disgusted, respectively. Previous research has shown that 
individuals oriented towards short-term mating are less disgusted (Al-Shawaf et al., 2019; Crosby et al., 2020; Tybur et al., 2009). It is important to control for these potential confounds, so that we do not artificially inflate the association between perceptions of mate availability and sexual disgust. We asked participants to indicate if they were in a relationship with a dichotomous "yes" or "no" question. To assess participants' mating orientation, we asked them to indicate how much they agreed ( $1=$ strongly disagree; $2=$ somewhat disagree; $3=$ somewhat agree; $4=$ strongly agree) with two statements about the extent to which they are currently seeking a short-term, uncommitted relationship or a long-term, committed relationship ("I am currently seeking shortterm, uncommitted relationships (e.g., casual sex, one-night stands, brief affairs); "I am currently seeking a long-term, committed relationship (e.g., a committed romantic relationship or marriage)".

Attention and validity checks. At two points in the survey-once during the self-perceived attractiveness scale, and once during the self-perceived mate value scale-we asked participants to select a specific rating scale option to ensure that they are paying attention. At the completion of the survey, we also asked participants to indicate how carefully they paid attention, and how honest they were throughout the survey (using a continuous sliding scale ranging from 0-100\%). Participants were assured that their answers to these questions would not affect their compensation in any way, but that their answers would simply be used to conduct more accurate analyses. We used forced responding to ensure that participants responded to all of the survey questions. If participants wished to stop responding for any reason, they were able to exit the survey at any time. We excluded participants from confirmatory analyses if they failed to correctly answer both attention check questions or if they reported that they were not paying attention and responding honestly to at least $80 \%$ of the survey.

\section{Procedure}

Participants were recruited through Prolific, our University's psychology subject pool, and snowball sampling through social media platforms (e.g., Facebook, Twitter, Reddit) to participate in an online study about emotions. If participants agreed to take part in our study, they were directed to fill out an online survey through the online survey-hosting software Qualtrics.

Participants first granted informed consent and completed demographics questions. In order to assign participants to rate faces of the appropriate sex, participants then indicated which gender they are most attracted to when it comes to selecting a sexual partner. Participants who reported preferring men rated all 60 male faces, participants who reported preferring women rated all 60 female faces, and participants who reported no preference rated a random subset of 60 faces of either sex. The faces were presented in a random order for each participant.

Participants were randomly assigned to one of two conditions: Condition A required participants to first answer questions about their self-perceived mate 
availability and subsequently rate faces, and Condition B required participants to first rate faces and subsequently fill out questions about their self-perceived mate availability. Doing this allowed us to explicitly test and control for potentially confounding demand characteristics and order effects. After completing 60 face ratings and the self-perceived mate availability questionnaire, participants answered questions about their self-perceived mate value, attractiveness, control questions, and the attention and honesty questions. Participants were then debriefed, thanked for participation, and provided with a link to be compensated if they were participating through Prolific.

\section{Power Analysis}

Prior to data collection, we conducted power simulations using $\mathrm{R}$ to determine how many participants we would need to collect, given that our stimuli sample size is fixed. We modified code provided by DeBruine and Barr (2019) to create power analysis functions for our specific needs. The simulated data sets cross raters with targets of the opposite sex and create an outcome variable (e.g., sexual disgust) based on both rater- and target-level continuous (e.g., target attractiveness) and categorical (rater sex) covariates, in addition to rater- and target-level random variance. We describe the details of the power analyses below and the full code and simulation results are available to view on the OSF (see Power Simulations code on OSF).

The random-effects structure was held constant for all simulations: we specified large amounts of variability in target- and rater-level random intercepts, a moderate amount of variability in rater-level slopes, a moderate correlation between rater-level random slopes and intercepts, and a large residual error. These random-effects guesstimates are based on random-effect estimates from similar studies the authors have conducted where raters rate targets on various dimensions.

We specified effect size estimates for each fixed-effect of interest representing the smallest effect that would be theoretically interesting for our four primary hypotheses: (H1) a small positive association $(r=0.1)$ between raters' self-perceived mate availability (SPMA) and average sexual disgust across raters; (H2) a moderate sex difference $(d=0.4)$ in raters' average sexual disgust across targets; (H3) a moderate negative association $(r=0.4)$ between targets' objective attractiveness and raters' disgust ratings; and (H4) a small interaction $(r=0.05)$ between raters' SPMA and targets' attractiveness, such that attractiveness has a slightly larger association at higher levels of SPMA than at lower levels of SPMA.

We estimated the power to detect each effect of interest across nine sample size conditions (100, 200, 300, 400, 500, 700, 800, and 900 raters). Each sample size condition tested the effects of interest across 1,000 simulated datasets. The results of the power analyses are shown in Fig. 1. Unsurprisingly, the effect that requires the most raters to achieve $90 \%$ power is the small interaction between SPMA and targets' third-party rated attractiveness. 


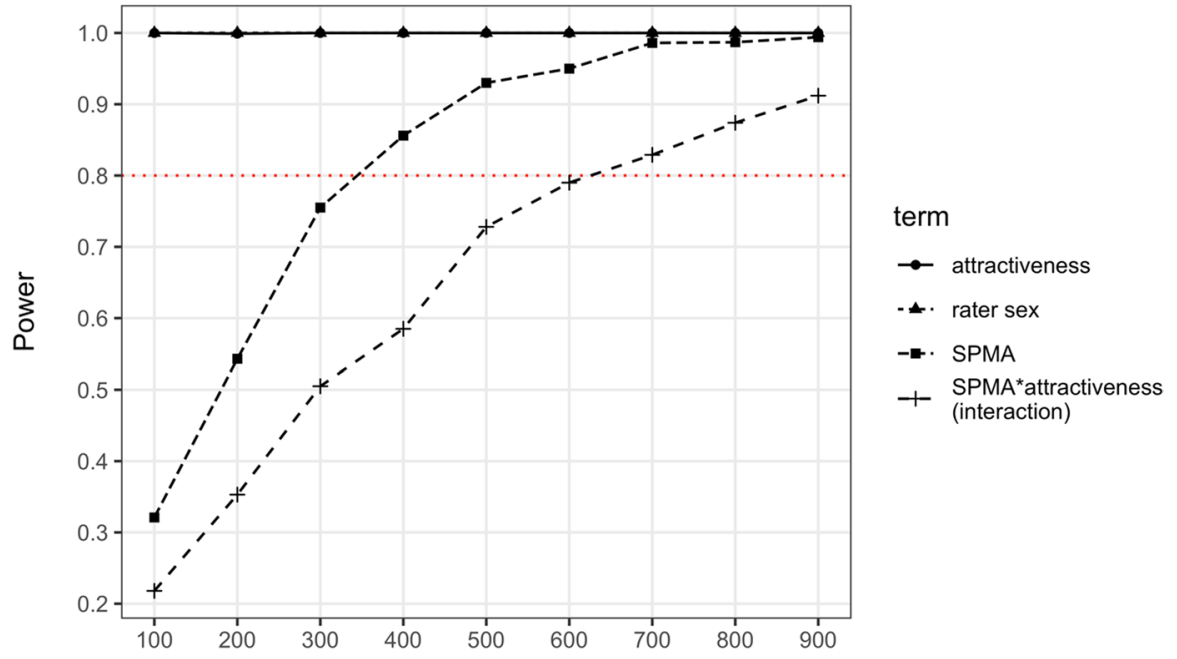

Rater Sample Size

Fig. 1 Plot of power curves, depicting the power to detect each effect of interest at various sample sizes, given the other constant parameters in the model. The power analyses indicated that we needed $90 \%$ power to detect the smallest of our hypothesized associations (i.e., the interaction between mate availability perceptions and target attractiveness) with 900 raters

\section{Results}

\section{Preregistered Analyses}

We used linear mixed effect models (LMEMs) to test our hypotheses because of the nested structure of the data we collected. Maximal random effects structures were specified for all models, where participant intercepts and slopes as well as stimulus intercepts and slopes can vary where possible (Barr et al., 2013). Specifically, targets intercepts were allowed to vary in all models, and rater intercepts and slopes were allowed to vary in all models. We describe the confirmatory and exploratory models below and each are clearly specified in our analysis code (see Data Cleaning and Analysis Code).

Confirmatory analyses. Sexual disgust levels of each participant towards the faces was the outcome variable. We regressed these sexual disgust ratings on (1) effect-coded rater sex, where the value of 1 is given to men and -1 is given to women; (2) the scaled average third-party ratings of targets' attractiveness; (3) scaled raters' perceptions of mate-availability; (4) an interaction term between targets' attractiveness and raters' self-perception of mate availability; and (5) the control variablesscaled raters' self-perceived mate value and attractiveness, effect-coded relationship status (-1=“no", 1 = “yes"), scaled continuous short-term mating orientation, scaled 
continuous long-term mating orientation, rater age, and categorical rater ethnicity ${ }^{6}$ and target ethnicity.

Figure 2 depicts the results of our focal hypotheses. Contrary to our prediction that perceived mate availability would be positively associated with sexual disgust (H1), perceived mate availability was negatively associated with baseline levels of sexual disgust across potential mates $(\beta=-0.05, p=0.022)$. Further, although we predicted that women's sexual disgust would be higher across potential mates than men's $(\mathrm{H} 2)$, we found no significant sex difference in levels of sexual disgust between men and women $(\beta=0.01, p=0.730)$. We did, however, find the predicted negative association between participants' sexual disgust and targets' facial attractiveness $(\beta=-0.46, p=2 \mathrm{e}-16)$ such that more attractive potential mates were considered less sexually disgusting (H3). And finally, the interaction between target attractiveness and mate availability perceptions on levels of sexual disgust was statistically significant $(\beta=-0.03, p=1.96 \mathrm{e}-4)$ as predicted (H4) such that individuals who perceived there to be many mates in their local environment weighted the attractiveness of potential mates slightly more heavily and tended to be more disgusted by less-attractive individuals than did individuals who perceived there to be few available mates in their local environment.

Sensitivity analyses. We examined potential demand characteristics and order effects by including a three-way interaction between a dichotomous "rating condition" term (indicating whether the participant rated faces before or after rating SPMA), target attractiveness, and raters' self-perceived mate availability. Because the three-way interaction was not significant $(\beta=0.003, p=0.677)$, we examined the two-way interactions between the three variables. The two-way interaction between rating condition and target attractiveness was not significant $(\beta=-0.03$, $p=0.100$ ), nor was the two-way interaction between rating condition and SPMA $(\beta=0.003, p=0.839)$. Next, we removed all interaction terms to examine if there was a simple effect of rating condition on disgust ratings. Results revealed no significant main effect of rating condition on disgust ratings $(\beta=-0.03, p=0.139)$. All of these exploratory models contained the same control variables as the confirmatory models (see Data Cleaning and Analysis code). In sum, our confirmatory results appear robust to any potential demand characteristics or order effects introduced by our methods.

\footnotetext{
6 We note that we did not specify a reference group for the rater ethnicity variable in our preregistration. Because the default settings of R would have set the default reference group to "American Indian", which comprised less than $1 \%$ of the total sample, we grouped individuals who self-selected "American Indian" as their ethnicity with the those who self-selected "Other" as their ethnicity category and let R default to setting the reference group alphabetically, which led to the reference group being raters who self-selected "Asian" for their ethnicity. Analyses that do not collapse "American Indian" into "Other" are available on the OSF.
} 


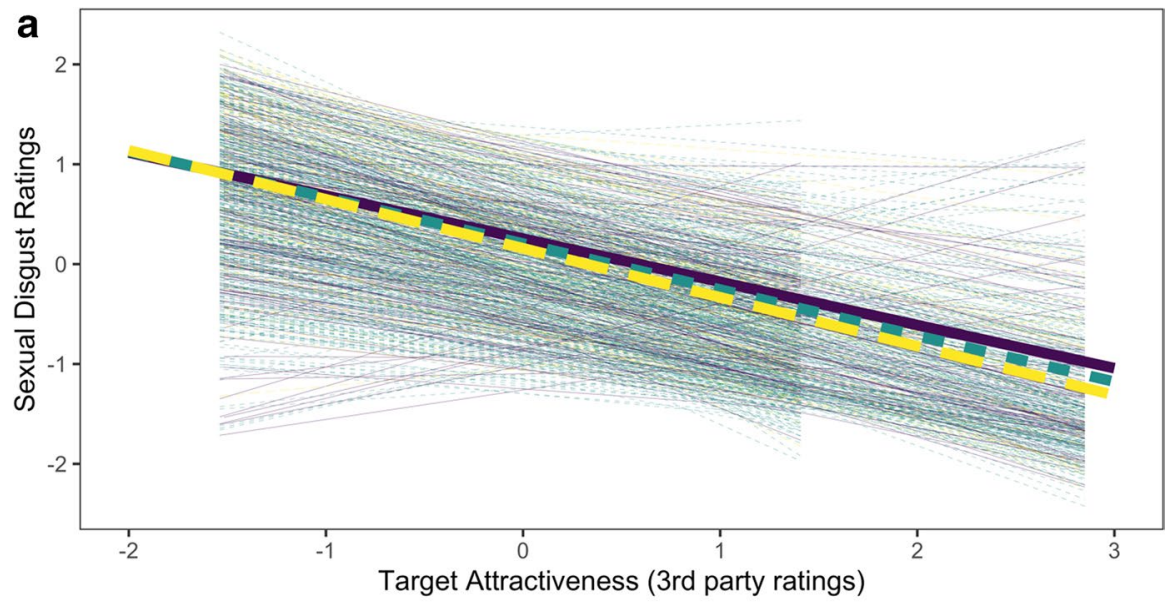

Low SPMA $=$ Ave SPMA High SPMA

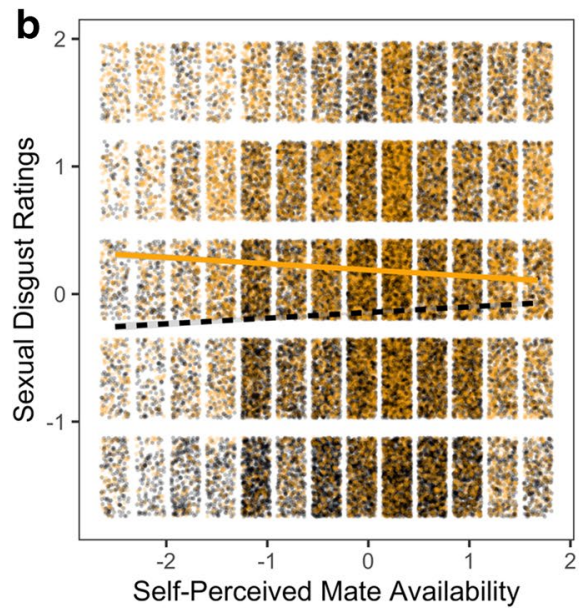

Female - - - Male

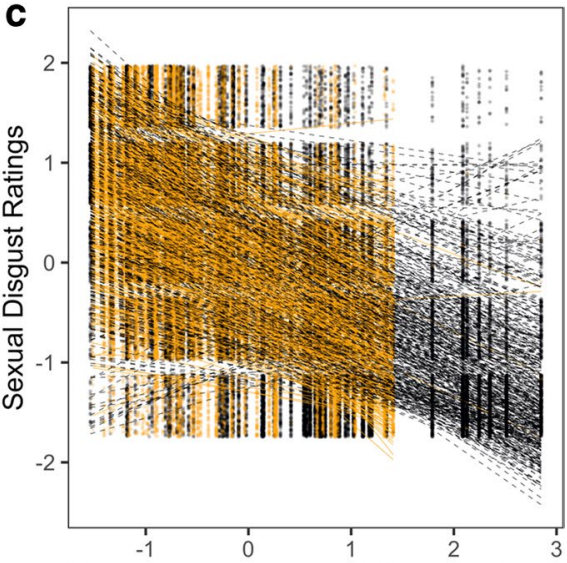

Target Attractiveness (3rd party ratings)

Female ...... Male

Fig. 2 Results of preregistered analyses in the final sample of 853 raters and 120 targets. Panel a depicts a small, significant interaction between raters' self-perceptions of mate availability (SPMA), the association between targets' third-party rated attractiveness, and raters' sexual disgust. Panel b depicts a small, significant negative association between raters' SPMA and sexual disgust. Panel c depicts a moderate, significant negative association between raters' sexual disgust and third-party ratings of targets' attractiveness 


\section{Exploratory Analyses}

Although our preregistered analysis found no statistically significant difference between men's and women's sexual disgust towards potential mates, we noticed upon visual inspection of our results in Fig. 2 that male targets were rated as consistently less attractive by the third-party raters than were female targets (Panel C). A post-hoc $t$-test revealing a large sex difference in target attractiveness supports this observation ( $t=4.4015, d f=104.43, p=2.60 \mathrm{e}-05$; Cohen's $d=0.80,95 \%$ CI [0.45, 1.19]). Unfortunately, we overlooked this in our preregistration plan.

The sex difference in third-party ratings of target attractiveness is important because if attractiveness ratings are related to disgust ratings (which our main analyses show), the fact that male targets were likely perceived as significantly less attractive by female raters than were female targets by male raters means that any effect of rater sex on sexual disgust levels in this study may be confounded with the third-party pre-rated attractiveness of the targets; so the effect of rater sex on sexual disgust ratings could be fully mediated by targets' third-party attractiveness ratings. In other words, rather than simply having an inherently less attractive sample of male photos, higher average female psychological sexual disgust levels might be the cause of the low third-party ratings of male photos, so the sex difference in disgust might be "baked into" the attractiveness ratings. Alternatively, women's perceptions of attractiveness and their lower average levels of sexual disgust may be driven by the same factor, such as women's greater selectivity in mating contexts (Buss \& Schmitt, 1993). Thus, including both terms in the model could wipe out the effect of any sex difference in disgust.

To examine whether the sex difference in third-party ratings of target attractiveness might account for the null effect of rater sex on sexual disgust initially uncovered in this study, we removed the attractiveness ratings from the full mixed effect model. When doing this, we found that male participants were indeed statistically less disgusted than female participants towards potential targets, both with the inclusion of the other control variables $(\beta=-0.15, p=5.56 \mathrm{e}-4)$ and without $(\beta=-0.15$ $p=3.52 \mathrm{e}-4)$. Thus, it appears that women did tend to be more sexually disgusted than men across potential mates, but this effect was confounded by the fact that male targets were perceived by a separate set of female raters as less attractive on average than female targets.

We also noticed in Fig. 2B that the association between self-perceived mate availability and sexual disgust appeared to differ between men and women. We therefore conducted post-hoc tests of this potential interaction by adding it to the full preregistered model. The interaction was significant $(\beta=0.06, p=9.72 \mathrm{e}-4)$, and decomposition showed that the relationship between perceived mate availability and sexual disgust was statistically significant and negative for women raters $(\beta=-0.11$, $p=2.32 \mathrm{e}-4)$ but not statistically significant for men $(\beta=0.01, p=0.737)$.

Finally, we explored the extent to which our results might be different had we not used such stringent exclusion criteria, which reduced our effective sample size by more than half. We re-ran all the preregistered and exploratory analyses described above without removing participants for failing attention checks, responding 


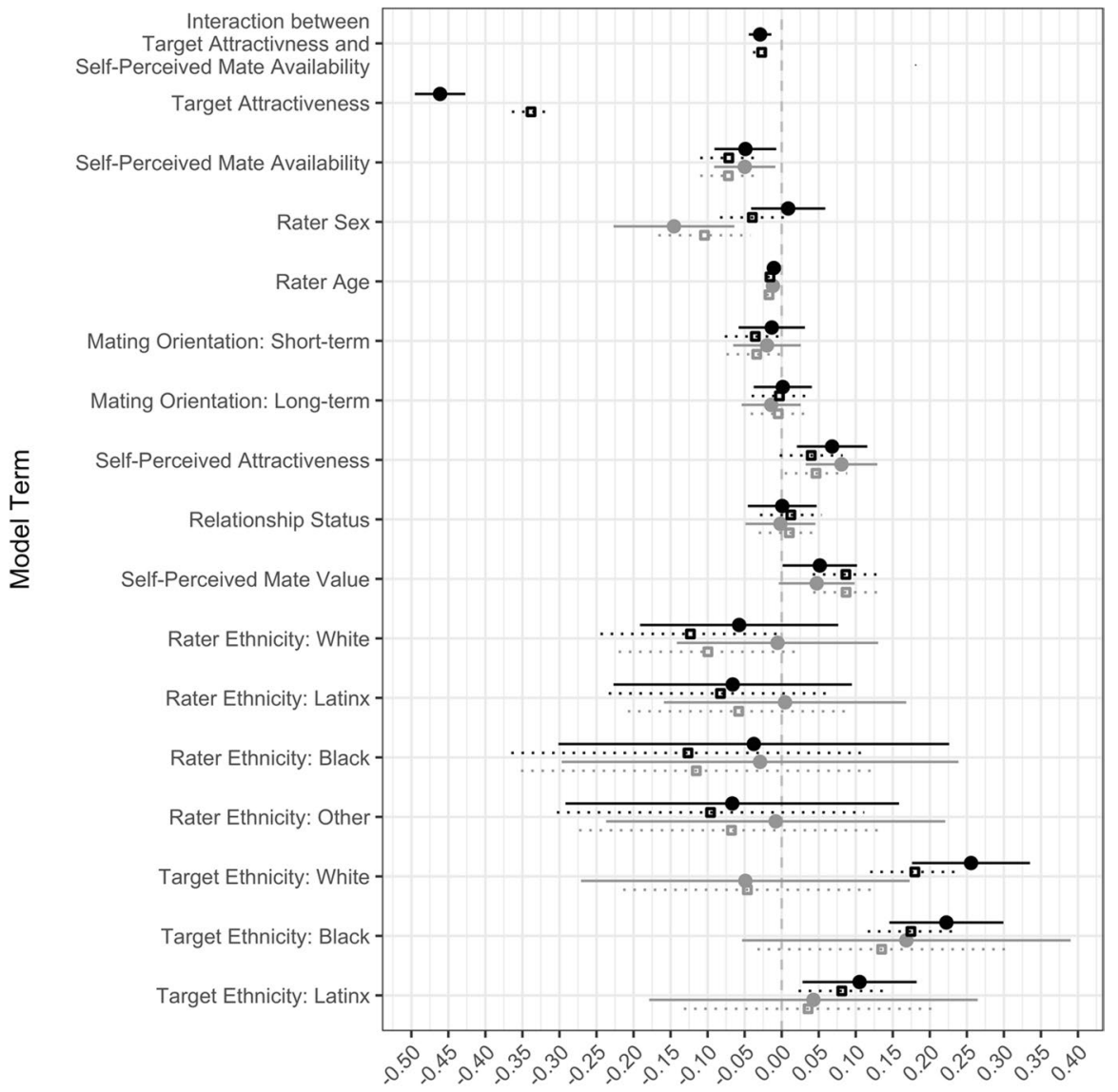

Association with sexual disgust ratings
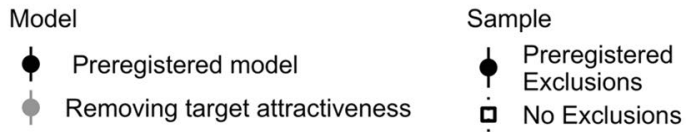

Fig. 3 Coefficient plot depicting the point estimates and 95\% confidence intervals for associations between sexual disgust ratings and different predictors to compare the results from the preregistered analysis (dark black color) to the exploratory analysis removing target attractiveness (lighter grey color), and comparing results using the preregistered exclusion criteria (filled circles and solid lines) and without exclusion criteria (empty squares and dotted lines). All associations reflect standardized associations except for the ethnicity variables which are dummy coded. The sample size under the pre-registered exclusion criteria is $\mathrm{N}=853$ (373 women); without exclusion criteria $\mathrm{N}=1,757$ ( 828 women)

carelessly, or self-reporting invalid responses. In this larger sample $(N=1,757$; 828 women), our results are qualitatively unchanged: none of the focal effects that were statistically significant in the preregistered sample were non-significant in the larger exploratory sample and effect sizes varied only superficially (see full output of the results with minimal participant exclusions on the OSF). Figure 3 presents 
an overview of the coefficients and $95 \%$ confidence intervals from the focal confirmatory and exploratory models we conducted, in order to facilitate comparison between models and samples.

\section{Discussion}

We examined the relationship between perceived mate availability and sexual disgust in this preregistered study. We hypothesized that (H1) perceived mate availability would be positively associated with sexual disgust across potential mates; (H2) women would be more sexually disgusted on average than men towards potential mates; (H3) attractiveness of potential mates would be inversely associated with sexual disgust; and (H4) sexual disgust towards potential mates would be more strongly associated with targets' attractiveness for raters who perceived there to be many available mates in their local mating pool than raters who perceived there to be fewer available mates.

As predicted, we found a negative association between sexual disgust and target attractiveness (H3), and a significant interaction between perceptions of mate availability and the association between targets' third-party rated attractiveness on raters' sexual disgust (H4). Our preregistered analyses did not support our prediction that women would report greater sexual disgust than men, on average, towards potential short-term mates (H2); however, exploratory analyses suggest that this effect may have been washed out by the confounding sex difference in third-party perceptions of attractiveness of male and female targets, as removing target attractiveness from the model revealed a small sex difference in sexual disgust levels in the predicted direction. Perhaps most notably among our findings, we found evidence contrary to our prediction about the association between mate availability and sexual disgust (H1): participants who perceived there to be few available mates in their local environment tended to have higher sexual disgust towards potential mates, but post-hoc analyses suggest this applies only to women.

Given that our results contradict our main hypothesis, what could explain the negative association between sexual disgust and mate availability uncovered in this study? While previous work suggests that mate availability may be an important contextual input into sexual disgust levels (Lieberman \& Patrick, 2018; Tybur et al., 2013), the causal direction could be reversed. Rather than conscious perceptions of mate availability driving sexual disgust levels, perhaps individuals who have lower baseline levels of sexual disgust consider more people as potential mates, increasing their perception of the size of the mate pool (Al-Shawaf et al., 2018). However, the sex difference in this relationship revealed by our exploratory analyses is perplexing. While there are well-established theoretical reasons to expect women to have higher sexual disgust on average than men (Al-Shawaf et al., 2018; Tybur et al., 2009), there is no clear reason to expect that men's sexual disgust should not influence the same internal regulatory variables (e.g., mate availability) that are influenced in women. As far as we know, the possibility that men's sexual disgust has less of an impact on their perceptions - and ultimately their sexual behavior-than women's has not yet been shown. On the contrary, recent research shows that while activating 
disgust leads to reduced interest in short-term mating for both sexes, there is no sex difference in the extent to which disgust manipulations impact desire for short-term mating (Al-Shawaf et al., 2019). If this effect is replicable, more research is necessary to untangle the sex difference in the association between sexual disgust and perceptions of mate availability.

It is likely that the null sex difference in sexual disgust initially found in this study exists because of the confounding effect of third-party ratings of target attractiveness. While it may intuitively appear that the sample of male photos used in this study was simply of lower mate quality than that of the female photos, it is worth considering the potential impact of evolved sex differences in sexual disgust on attractiveness ratings themselves. If women have higher sexual disgust levels than men, then third-party female raters would likely be more disgusted in response to the average male photo - regardless of mate quality — which may dampen perceptions of attractiveness. Indeed, when removing target attractiveness from the model, we found a small sex difference in sexual disgust in the predicted direction, replicating previous research (Al-Shawaf et al., 2018; Crosby et al., 2020; Tybur et al., 2009).

While our planned analysis did not account for the sex difference in third-party ratings of target attractiveness, the difference is not surprising in retrospect. Sex differences in ratings of the attractiveness of the opposite sex are not often reported in attractiveness studies, but trends like the one in our study have occasionally been reported as incidental findings in some peer-reviewed articles (e.g., Gladue \& Delany, 1990; Wood \& Brumbaugh, 2009) and data from dating websites (e.g., Rudder, 2014). Wood and Brumbaugh (2009) also found a restricted range in ratings from female participants (mirroring that observed in our data), which they attribute to women's greater general selectivity in mating (Buss \& Schmitt, 1993). However, the sex difference could be mediated by baseline sexual disgust levels, or a third variable such as sociosexual orientation-which tends to be less restricted in men-since physical attractiveness is weighted more heavily in short-term mating contexts (Li \& Kenrick, 2006). Future research should disentangle sex differences in target attractiveness, and the impact of sex differences in target attractiveness on sexual disgust towards potential mates in long-term mating contexts.

Because attractiveness is hypothesized to be an observable proxy for overall mate value and overall mate value is an input hypothesized to calibrate sexual disgust levels (e.g., Tybur et al., 2013), the inverse association uncovered between target attractiveness and disgust in this study is not particularly surprising. While attractiveness is a known component of overall mate value, previous work suggests that attractiveness may also trigger sexual desire (McCall \& Meston, 2006). This possibility is important to consider because researchers hypothesize that sexual disgust may exist on a continuum of sexual motivation, with sexual desire-or lust-being the opposite of sexual disgust (Lieberman \& Patrick, 2018). These two motivational processes can intuitively be thought of as two ends of a spectrum-one motivating sexual approach and the other motivating sexual avoidance. While we do not have evidence to directly examine the comparative computational structure of sexual desire and sexual disgust in this study, the negative correlation between attractiveness and disgust suggests that this may be an accurate 
conceptualization of these two motivational processes (per Lieberman \& Patrick, 2018). If sexual disgust and sexual desire truly exist on a continuum, then changes in cues associated with disgust activation in a partner (e.g., sores or lesions) may impact the perceived attractiveness and reported rates of sexual desire towards the partner. Extant research has not directly examined this possibility, but disfigured faces have been shown to elicit disgust (e.g., Stone \& Potton, 2019). Future researchers may consider experimentally manipulating the disgust cues of potential mates and subsequently observing how these manipulations impact the perceived attractiveness and desirability of targets in short and long-term mating contexts.

\section{Limitations}

This study has several limitations that should be considered. First, we did not achieve our preregistered sample size of 900 participants because we lost an unexpectedly large number of participants to our stringent exclusion criteria. As a result, our study may be slightly underpowered-although, our sample size is more than double that of previous work (see Arnocky, 2018; Arnocky et al., 2014, 2016). Further, according to our simulated power analyses (see Fig. 1), a sample size of 800 raters provides more than $80 \%$ power to detect the smallest of our hypothesized associations (i.e., the interaction between mate availability perceptions and target attractiveness). Importantly, our post-hoc analyses based on less restrictive exclusion criteria providing more than $90 \%$ power to detect the predicted effects did not show any qualitative differences in the results.

Second, there may be an important functional distinction between the perceived number of possible mates and the perceived number of desirable or feasible mates. We took this possible distinction into account when designing the scale questions to assess participants' perceptions of available mates. However, it is possible that the methodology employed in this study did not adequately disentangle the two.

Finally, all of the data for this study was collected during the COVID-19 pandemic. Our first round of data collection began in June 2020, and our last round ended in January of 2021. Although we argue that there are potential costs to our perception psychology being easily manipulated by things like newspaper articles and fabricated photos, it is possible that COVID-19-related policies-and the social changes they bring about-represent the kinds of cues that our psychology may be calibrated to. Failing to recalibrate disgust levels in response to cues associated with a novel, easily transmittable, life-threatening disease could present numerous costs-reputationally, medically, and psychologically. COVID-19-related policies such as social distancing, wearing masks, and quarantining may be tied to unique variation in perceptions of mate availability, disgust, and our mating psychology more broadly during this time. While the impact of the COVID-19 pandemic on our underlying sexual psychology remains unclear, it is important to keep this limitation in mind. 


\section{Future Directions}

If sexual disgust is used as an input to perceived mate availability, it should be relatively simple to demonstrate. To do so, future studies could manipulate sexual disgust and subsequently measure perceived mate availability. In addition, our initially proposed mechanism of mate availability calibrating sexual disgust levels could be categorically ruled out if a complementary experiment manipulating perceived mate availability changed subsequent levels of sexual disgust. Such experiments would help tease apart the internal regulatory variables involved in mate-choice decisions.

Since this study could not account for all possible inputs to both sexual disgust and perceived mate availability, it is also possible that there are other variables involved. Another internal regulatory variable — such as perceived genetic relatedness-might raise disgust levels, eliminating the lower echelon of potential mates from the pool of genuine mating prospects, thus indirectly reducing the perceived size of the mating pool as a function of desirability. Alternatively, this effect may not be a byproduct. The mate availability internal regulatory variable could be functionally dissociated from reality in order to alter future internal calculations in favor of behaving as if the mating pool were actually smaller. The distinction between nominal and pragmatic mate availability could be essential in understanding our relevant information-processing mechanisms. Future research should consider other possible factors that could be relevant to calculations of the number of people that are genuine mating prospects.

More insight could also be gained by improving methods of measuring actual mate availability. If our reversed-causality explanation for the negative association between sexual disgust and perceptions of mate availability is correct, the accuracy of our conscious perception of mate availability must be scrutinized more closely. Operational sex ratio may be a crude proxy at best, as it may fail to take into account many important factors that would likely be tracked by our minds, such as age distribution, proportion of mates that are married or pregnant, and local attitudes toward monogamy and casual sex (see Brandner et al., 2020 for a discussion of our evolved sex ratio tracking abilities). More effort should be dedicated to expanding the range of measures used to approximate actual mate availability.

\section{Conclusion}

Theoretical considerations suggest that the number of available mates in a given environment should influence sexual disgust levels. This study tested this hypothesis by investigating the association between perceptions of mate availability and sexual disgust towards potential short-term mates. The results suggest that sexual disgust may not be calibrated to the size of the mate pool as hypothesized. Rather, individuals with lower baseline levels of sexual disgust may perceive there to be more mates to select from and, as a result, have a mental representation of a larger mate pool.

\section{Declarations}

Conflict of Interests The authors declare that they have no conflict of interest. 


\section{References}

Ackerman, J. M., Kenrick, D. T., \& Schaller, M. (2007). Is friendship akin to kinship? Evolution and Human Behavior, 28, 365-374.

Al-Shawaf, L., Lewis, D. M., Ghossainy, M. E., \& Buss, D. M. (2019). Experimentally inducing disgust reduces desire for short-term mating. Evolutionary Psychological Science, 5(3), 267-275.

Al-Shawaf, L., Lewis, D. M., \& Buss, D. M. (2018). Sex differences in disgust: Why are women more easily disgusted than men? Emotion Review, 10(2), 149-160.

Al-Shawaf, L., Conroy-Beam, D., Asao, K., \& Buss, D. M. (2016). Human emotions: An evolutionary psychological perspective. Emotion Review, 8(2), 173-186.

Al-Shawaf, L., Lewis, D. M., \& Buss, D. M. (2015). Disgust and mating strategy. Evolution and Human Behavior, 36(3), 199-205.

Arnocky, S. (2018). Self-perceived mate value, facial attractiveness, and mate preferences: Do desirable men want it all? Evolutionary Psychology, 16(1), 1474704918763271.

Arnocky, S., Woodruff, N., \& Schmitt, D. P. (2016). Men's sociosexuality is sensitive to changes in mate availability. Personal Relationships, 23(1), 172-181.

Arnocky, S., Ribout, A., Mirza, R. S., \& Knack, J. M. (2014). Perceived mate availability influences intrasexual competition, jealousy and mate-guarding behavior. Journal of Evolutionary Psychology, 12(1), 45-64.

Barr, D. J., Levy, R., Scheepers, C., \& Tily, H. J. (2013). Random effects structure for confirmatory hypothesis testing: Keep it maximal. Journal of Memory and Language, 68(3), 255-278.

Brandner, J. L., Dillon, H. M., \& Brase, G. L. (2020). Convergent evidence for a theory of rapid, automatic, and accurate sex ratio tracking. Acta Psychologica, 210, 103161.

Buss, D. M., \& Shackelford, T. K. (2008). Attractive women want it all: Good genes, economic investment, parenting proclivities, and emotional commitment. Evolutionary Psychology, 6(1), 147470490800600130 .

Buss, D. M., \& Schmitt, D. P. (1993). Sexual strategies theory: An evolutionary perspective on human mating. Psychological Review, 100(2), 204.

Buss, D. M. (1989). Sex differences in human mate preferences: Evolutionary hypotheses tested in 37 cultures. Behavioral and Brain Sciences, 12(1), 1-14.

Cosmides, L., \& Tooby, J. (2000). Evolutionary psychology and the emotions. Handbook of Emotions, 2(2), 91-115.

Crosby, C. L., Durkee, P. K., Meston, C. M., \& Buss, D. M. (2020). Six dimensions of sexual disgust. Personality and Individual Differences, 156.

Curtis, V., Aunger, R., \& Rabie, T. (2004). Evidence that disgust evolved to protect from risk of disease. Proceedings of the Royal Society of London B: Biological Sciences, 271(Suppl 4), S131-S133.

Daly, M., \& Wilson, M. (2001). Risk-taking, intrasexual competition, and homicide. In J. A. French, A. C. Kamil, \& D. W. Leger (Eds.), Vol. 47 of the Nebraska symposium on motivation. Evolutionary psychology and motivation (pp. 1-36). University of Nebraska Press.

DeBruine, L., \& Barr, D. J. (2019). Understanding mixed effects models through data simulation.

Fisher, M., Cox, A., Bennett, S., \& Gavric, D. (2008). Components of self-perceived mate value. Journal of Social, Evolutionary, and Cultural Psychology, 2(4), 156.

Gladue, B. A., \& Delaney, H. J. (1990). Gender differences in perception of attractiveness of men and women in bars. Personality and Social Psychology Bulletin, 16(2), 378-391.

Griskevicius, V., Tybur, J. M., Ackerman, J. M., Delton, A. W., Robertson, T. E., \& White, A. E. (2012). The financial consequences of too many men: Sex ratio effects on saving, borrowing, and spending. Journal of Personality and Social Psychology, 102(1), 69.

Hahn, A. C., Fisher, C. I., DeBruine, L. M., \& Jones, B. C. (2014). Sex ratio influences the motivational salience of facial attractiveness. Biology Letters, 10(6), 201401.

Haidt, J., Bjorklund, F., \& Murphy, S. (2000). Moral dumbfounding: When intuition finds no reason. Unpublished manuscript, University of Virginia.

Jones, B. C., DeBruine, L. M., Flake, J. K., Liuzza, M., Antfolk, J., Arinze, N. C., ... Coles, N. A. (2018). To Which World Regions Does the Valence-Dominance Model of Social Perception Apply?. https:// doi.org/10.31234/osf.io/n26dy

Landolt, M. A., Lalumière, M. L., \& Quinsey, V. L. (1995). Sex differences in intra-sex variations in human mating tactics: An evolutionary approach. Ethology and Sociobiology, 16(1), 3-23. 
Li, N. P., \& Kenrick, D. T. (2006). Sex similarities and differences in preferences for short-term mates: What, whether, and why. Journal of Personality and Social Psychology, 90(3), 468.

Lieberman, D., \& Patrick, C. (2018). Objection: disgust, morality, and the law. Oxford University Press.

Little, A. C., Jones, B. C., \& DeBruine, L. M. (2011). Facial attractiveness: Evolutionary based research. Philosophical Transactions of the Royal Society B: Biological Sciences, 366(1571), 1638-1659.

Ma, D. S., Correll, J., \& Wittenbrink, B. (2015). The chicago face database: A free stimulus set of faces and norming data. Behavior Research Methods, 47, 1122-1135.

McCall, K., \& Meston, C. (2006). Cues resulting in desire for sexual activity in women. The journal of sexual medicine, 3(5), 838.

Nesse, R. M. (1990). Evolutionary explanations of emotions. Human Nature, 1(3), 261-289.

Ryan, S., Oaten, M., Stevenson, R. J., \& Case, T. I. (2012). Facial disfigurement is treated like an infectious disease. Evolution and Human Behavior, 33(6), 639-646.

Rudder, C. (2014). Dataclysm: Love, Sex, Race, and Identity--What Our Online Lives Tell Us about Our Offline Selves. Crown.

Stone, A., \& Potton, A. (2019). Emotional responses to disfigured faces and disgust sensitivity: an eyetracking study. Journal of Health Psychology, 24(9), 1191-1200.

Thornhill, R., \& Gangestad, S. W. (1999). Facial attractiveness. Trends in Cognitive Sciences, 3(12), $452-460$.

Tybur, J. M., Lieberman, D., Kurzban, R., \& DeScioli, P. (2013). Disgust: Evolved function and structure. Psychological Review, 120(1), 65.

Tybur, J. M., Lieberman, D., \& Griskevicius, V. (2009). Microbes, mating, and morality: Individual differences in three functional domains of disgust. Journal of Personality and Social Psychology, 97(1), 103.

Watkins, C. D., Jones, B. C., Little, A. C., DeBruine, L. M., \& Feinberg, D. R. (2012). Cues to the sex ratio of the local population influence women's preferences for facial symmetry. Animal Behaviour, $83(2), 545-553$.

Wood, D., \& Brumbaugh, C. C. (2009). Using revealed mate preferences to evaluate market force and differential preference explanations for mate selection. Journal of Personality and Social Psychology, 96(6), 1226.

Yong, J. C., \& Li, N. P. (2012). Cash in hand, want better looking mate: Significant resource cues raise men's mating standards. Personality and Individual Differences, 53(1), 55-58.

Publisher's Note Springer Nature remains neutral with regard to jurisdictional claims in published maps and institutional affiliations. 